\title{
CORTICAL INTEGRATIVE THERAPY FOR THE TREATMENT OF MIGRAINES AND HEADACHES
}

\author{
Nicole C. Lim ${ }^{1}$, Victor M. Pedro ${ }^{1,2}$ and Elena Oggero ${ }^{3,4}$ \\ ${ }^{1}$ iBrain - International Institute for the Brain, New York, NY \\ ${ }^{2}$ Director of Rhode Island Integrated Medicine, Cranston, RI \\ ${ }^{3}$ Electrical and Computer Engineering Department, University of Wyoming, Laramie, WY \\ ${ }^{4}$ Vestibular Technologies, LLC, Cheyenne, WY
}

Corresponding Author: Victor M. Pedro

International Institute for the Brain - iBRAINnyc.org - 311 East 94th St. New York, NY 10128, USA

Tel: +1 646-315-1548

Email: vpedro@iBRAINnyc.org

https://doi.org/10.34107/BiomedSciInstrum.57.04168

\begin{abstract}
Migraine is a common neurological disorder that is characterized by a host of symptoms including severe throbbing headaches. In this retrospective chart review, the effectiveness of Cortical Integrative Therapy (PedroCIT®) was examined in adults with migraines. Multivariate General Linear Model (M-GLM) was utilized to determine if the emotional, functional, and overall difficulties, as well as the intensity of pain experienced with headaches decreased from before to after PedroCIT® treatment in individuals with mild to complete disability resulting from headaches. Repeated Measures General Linear Model (RM-GLM) was also used to investigate if postural stability increased from pre- to post-treatment. The results of the M-GLM showed that PedroCIT ${ }^{\circledR}$ was effective in reducing emotional, functional, overall disability, and intensity of pain resulting from headaches. Furthermore, RM-GLM indicated that patients who underwent PedroCIT ${ }^{\circ}$ improved their postural stability from pre- to post-treatment. Finally, the findings also showed that the duration of the treatment did not have any effect among patients with varied degrees of headache disability. This study illustrates the effectiveness of PedroCIT ${ }^{\circledR}$ in the treatment of headaches and postural instability in migraine patients.
\end{abstract}

Keywords: Practice-based clinical research, Cortical integrative therapy, Computerized dynamic posturography, Headache disability index, Migraines and headaches, Numerical pain rating scale

\section{INTRODUCTION}

Migraine is a brain disorder that affects between $15.9 \%$ to $16.6 \%$ of the population in the United States of America [1-2]. Population studies have shown sex and age-related risk factors wherein females and certain age groups (e.g., individuals between the ages of 30 to 39 years) were disproportionately more impacted by migraines than their counterparts [1, 3-4].

Migraine is a disabling condition that is characterized by severe, pulsating headaches that can affect either one or both sides of the head. Other symptoms of migraine include nausea, vomiting, light and sound sensitivity, and loss of concentration [5-6]. Migraines may be presented with or without auras, with the latter being more common [7]. Auras are visual and sensory disturbances that can occur before or accompany a migraine episode. These visual and sensory experiences include photopsia, loss of vision, feelings of numbness, paresthesia, or difficulty with speech [5,8]. Migraines can also be classified as episodic or chronic, whereby episodic migraines involve having headaches between 0 to 14 days per month, while chronic migraines involve having headaches for at least 15 days per month for 3 months or longer [9]. The symptoms of migraines can be so debilitating that it can hinder an individual's mental health, work productivity, and daily life. 